\title{
Somatic mosaicism in trichorhinophalangeal syndrome: a lesson for genetic counseling
}

\author{
Carole Corsini ${ }^{1}$, Martin Gencik ${ }^{2}$, Marjolaine Willems ${ }^{1}$, Eva Decker ${ }^{2}$, Elodie Sanchez ${ }^{1,3}$, Jacques Puechberty ${ }^{4}$, \\ Anouck Schneider ${ }^{4}$, Manon Girard ${ }^{4}$, Patrick Edery ${ }^{5}$, Patricia Bretonnes ${ }^{5}$, Jérôme Cottalorda ${ }^{6}$, \\ Geneviève Lefort ${ }^{4}$, Claire Jeandel ${ }^{7}$, Pierre Sarda ${ }^{1}$ and David Genevieve ${ }^{\star, 1,3}$
}

Trichorhinophalangeal syndrome type I (TRPSI) is a genetic disorder characterized by sparse hair, a bulbous nasal tip, short stature with severe generalized shortening of all phalanges, metacarpal and metatarsal bones and cone-shaped epiphyses. This syndrome is caused by autosomal dominant mutations in the TRPS1 gene. However, because recurrence has been observed in siblings from healthy parents, an autosomal recessive mode of inheritance has also been suggested. We report on a male patient, born to healthy unrelated parents, with TRPSI. Using Sanger sequencing, we identified a mutation in the TRPS1 gene (c.2735 G > A, P.Cys912Tyr). The same mutation was detected as a $10 \%$ mosaic mutation by Pyrosequencing in blood-derived DNA from his healthy mother. To our knowledge, this is the first time that somatic mosaicism has been identified in TRPSI. This data combined with the observations of recurrences in siblings from healthy parents modifies the genetic counseling for TRPSI, which should discuss a 5-10 percent recurrence risk for healthy parents with an affected child because of the possibility of germinal mosaicism.

European Journal of Human Genetics (2014) 22, 136-139; doi:10.1038/ejhg.2013.56; published online 10 April 2013

Keywords: autosomal dominant; autosomal recessive; genetic counseling; mosaicism; trichorhinophalangeal syndrome type 1; TRPS1 gene

\section{INTRODUCTION}

Trichorhinophalangeal syndrome (TRPS) type I (TRPSI, MIM 190350 ) is characterized by distinctive craniofacial features (sparse scalp hair, bulbous tip of the nose, long flat philtrum, thin upper vermilion border and protruding ears) and by skeletal abnormalities including cone-shaped phalangeal epiphyses, small hip epiphyses and short stature. The first patients with TRPSI were described by Klingmuller ${ }^{1}$ in 1956. Giedon ${ }^{2,3}$ coined the name trichorhinophalangeal syndrome in 1966. TRPS type II (TRPSII, MIM 150230), also called Langer-Giedon syndrome, results in a similar phenotype with the addition of intellectual deficiency and bony exostoses. TRPS type III (TRPSIII, OMIM 190351) differs clinically from TRPSI by the appearance of severe brachydactyly and extreme shortness of stature. ${ }^{4}$

TRPSI and III are caused by mutations in the TRPS1 gene, TRPSIII being most often caused by a specific class of mutations in exon 6 , whereas TRPSII is caused by $8 \mathrm{q} 23.3-\mathrm{q} 24.13$ microdeletion leading to the loss of at least two genes: TRPS1 and Exostosin 1 (EXT1, MIM 608177).

The zinc finger transcription factor gene (TRPS1, MIM 604386) is localized on chromosome $8 \mathrm{q} 24$ and encodes for a zinc finger transcriptional repressor involved in the regulation of chondrocyte and perichondrium development. ${ }^{5,6}$
Although the majority of reported kindreds with TRPSI are consistent with autosomal dominant inheritance, in some cases, autosomal recessive inheritance has been suggested. ${ }^{1,7-9}$

We report on a male patient presenting with TRPSI, the identification of TRPS1 mutation in the patient and the discovery of the same mutation, but in somatic mosaicism, in the healthy mother.

\section{MATERIALS AND METHODS}

\section{Patient}

The patient was 12 years old when he was referred to the Clinical Genetics Department for growth delay and facial features suggestive of TRPS1. He was the only child born to healthy unrelated parents without clinical features suggestive of TRPS1. The heights of the mother and the father were $160 \mathrm{~cm}$ and $180 \mathrm{~cm}$, respectively.

Prenatal screening revealed intrauterine growth retardation at 30 weeks of gestation (WG) with femur length shortening. No prenatal investigations were performed. The baby was born at $41 \mathrm{WG}$ and 3 days. Birth parameters showed only length below the normal range (birth weight: $2930 \mathrm{~g},-1.5$ standard deviation (SD); birth length: $46 \mathrm{~cm},-3 \mathrm{SD}$ and OFC: $34 \mathrm{~cm},-1 \mathrm{SD}$ ).

Motor development was normal. The boy presented speech delay, requiring speech therapy, a consequence of hearing impairment, secondary to otitis, but no intellectual disability. Postnatal growth was regular at -1 SD until the age of 2 years and then followed by deceleration of growth at -1.5 SD despite a normal parental height $(160 \mathrm{~cm}$ and $180 \mathrm{~cm}$ for the mother and the father, respectively). The patient later presented painful bilateral osteochondritis.

${ }^{1}$ Département de Génétique Médicale, Centre de Référence Maladies Rares Anomalies du Développement et Syndromes Malformatifs Sud-Languedoc Roussillon, CHRU Montpellier, Faculté de Médecine Université Montpellier 1, Montpellier, France; ${ }^{2}$ Diagenos, Center for Medical Genetics, Osnabrück, Germany; ${ }^{3}$ Unité INSERM U844, Institut des Neurosciences de Montpellier, Montpellier, France; ${ }^{4}$ Laboratoire de Génétique Chromosomique, Plate-forme puces à ADN, CHRU Montpellier, Faculté de Médecine Université Montpellier 1, Montpellier, France; ${ }^{5}$ Service de Génétique Clinique et Moléculaire, Hospices Civils de Lyon, Lyon, France; ${ }^{6}$ Département de Chirurgie Orthopédique Infantile, CHRU de Montpellier, Montpellier, France; ${ }^{7}$ Département de Pédiatrie Endocrinologique, CHRU de Montpellier, Montpellier, France

*Correspondence: Dr D Genevieve, Département de Génétique Médicale, Centre de Référence Maladies Rares Anomalies du Développement et Syndromes Malformatifs Sud-Languedoc Roussillon, CHRU Montpellier, Faculté de Médecine Université Montpellier 1, 371 Avenue du doyen Gaston Giraud, 34000 Montpellier, France. Tel: +334673365 64, Fax: +334673360 52; E-mail: d-genevieve@chu-montpellier.fr

Received 14 October 2012; revised 26 February 2013; accepted 28 February 2013; published online 10 April 2013 
Physical examination at the age of 9 years revealed sparse hair, a bulbous nasal tip, thin lips, protruding ears, small incisors, and short hands and feet (Figures 1a and b). Diagnosis of TRPS I was suspected on skeletal X-rays showing small and irregular femoral epiphyses, cone-shaped epiphyses with delayed carpal bone age and absence of bony exostose (Figures $1 \mathrm{c}$ and d). The hands (Figure 1e) and skeletal X-rays of the mother did not present suggestive features of TRPSI (Figures if and g).

Brain MRI, kidney and heart ultrasounds and endocrinological screening for growth hormone and thyroid function were normal. Peripheral blood karyotype, FISH using Aquarius TRPS1/EXT1 probes (8q23.3 chromosomal region-Cytocell Ltd, Cambridge, UK) and MLPA analysis (SALSA MLPA probemix P096 Mental Retardation-2, MRC-Holland, Amsterdam, the Netherlands) were normal in the patient, thus, excluding a deletion or a large duplication of the TRPS1 gene.

\section{Molecular study}

DNA was extracted from the patient and parent whole-blood samples using the QIAamp DNA Blood Maxi Kit (Qiagen, les Ulis, France), according to the manufacturer's protocol.

Direct sequencing of PCR products covering all protein-coding exons of the TRPS1 gene was performed in the patient. For the parents, only the analysis of exon 6 was performed. The PCR products were also analyzed by Roche Junior 454 pyrosequencing (Roche, Branford, CT, USA) to study the familial mutation.

\section{RESULTS}

Molecular analysis of the TRPS1 gene showed a heterozygous c.2735 G > A; (P.Cys912Tyr) mutation in the patient (NM_014112.2) (Figure 2a). In silico analysis (MutationTaster, PolyPhen2) predicted this mutation to be most likely damaging. Molecular analysis was normal in the father (Figure 2b). In the healthy mother, a lower fluorescence peak of adenine was identified at the same position as in the mutation observed in the child, suggesting of a somatic mosaicism. The mutation was confirmed using high coverage Roche Junior 454 pyrosequencing and was estimated at $\sim 10 \%$ (Figure $2 \mathrm{c}$ ). These data were highly suggestive of somatic mosaicism in the mother.

\section{DISCUSSION}

Momeni et al. ${ }^{5}$ identified, in 10 unrelated patients, six different nonsense mutations in the TRPS1 gene. The findings suggested that haploinsufficiency for this putative transcriptor factor causes TRPSI.

According to the literature, approximately half of TRPSI patients have mutations inherited in an autosomal dominant manner, while the other half are sporadic cases due to de novo mutation. ${ }^{10-13}$ Inheritance in TRPSI patients is frequently compatible with an autosomal dominant mode, ${ }^{14-18}$ but the data from some kindreds seems to favor autosomal recessive inheritance. ${ }^{1,7-9}$

Indeed, the patients described in 1956 by Klingmuller ${ }^{1}$ were two sisters, born to healthy parents, with hypotrichosis, bulbous nose, smooth skin, stretch marks, atypical ears, cone-shaped epiphyses on hands and feet and short stature. ${ }^{1}$ Two others siblings (a boy and his younger sister) with TRPSI and born to healthy parents were also reported in the literature by Van der Werff ten Bosch. ${ }^{7}$ They presented with short stature and abnormalities of the metacarpals and middle phalanges. Radiographs of the hands of their mother, two sisters and two brothers were all normal. No information was available for the
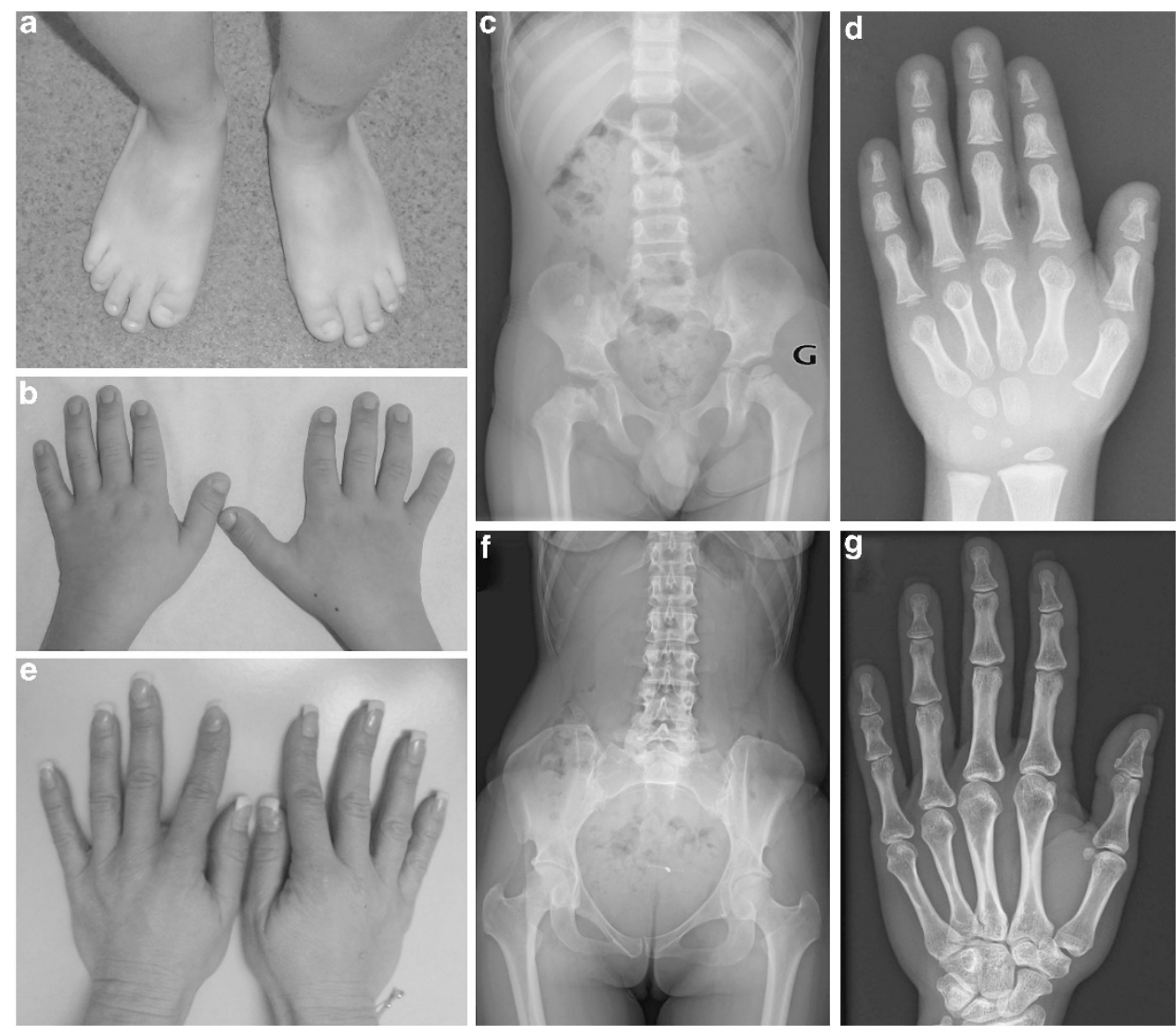

Figure 1 Clinical and radiological features in the patient and his mother. Note (a) and (b) brachydactyly, shortening of all phalanges (hands and feet) and fingers deviated at proximal interphalangeal joints, (c) femoral epiphyseal dysplasia, (d) cone-shaped epiphyses and carpal bone age delay (carpal bone age at 3 years for a chronological age of 5 years and 2 months) in the patient, and (e) normal hands, (f) normal femoral heads (g) and normal hands on the $\mathrm{X}$-rays of the mother. 


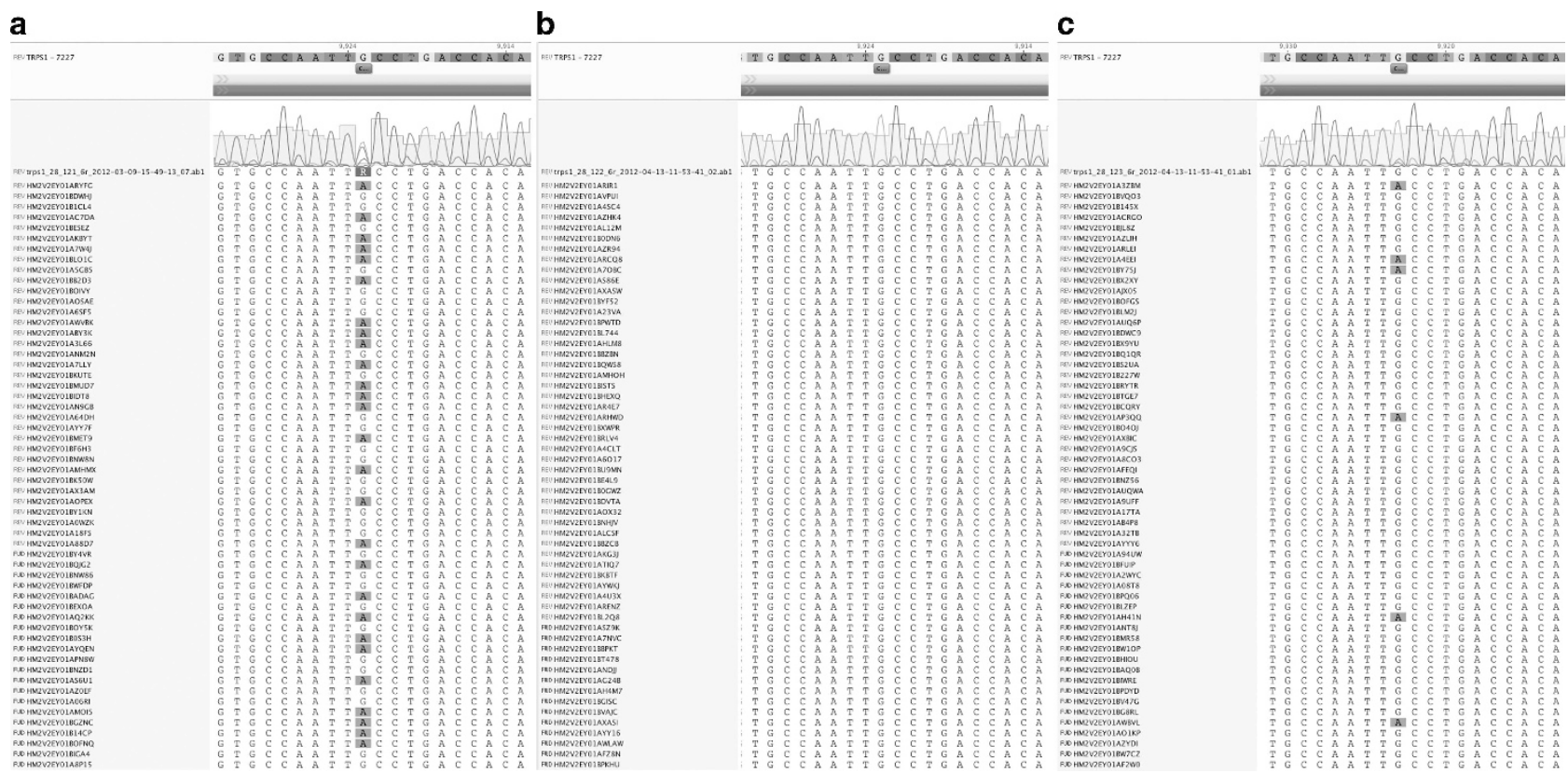

Figure 2 Molecular data from the patient and the healthy parents. Note (a) the heterozygous mutation c.2735G>A (P.Cys912Tyr) in exon 6 of the TRPS1 gene on the electropherogram and NGS data of the patient, (b) the familial mutation was not detected in the healthy father, (c) in the healthy mother, the c. $2735 \mathrm{G}>\mathrm{A}$ (P.Cys912Tyr) somatic mutation can be seen on the electropherogram and NGS data-note the mosaicism estimated at $\sim 10 \%$.

father. Giedion et al. ${ }^{18}$ concluded that autosomal recessive inheritance is the most likely explanation for these observations. ${ }^{1,7}$ Another family with three affected siblings was reported. The parents were not related and considered to be unaffected despite the fact that clinical examination of the father was not possible. ${ }^{8}$ In 1995 , a 10 -year-old girl with clinical characteristics of TRPSI was reported. One of her four siblings, a younger brother was thought to be affected because of increased density in some of the epiphyses of the distal phalanges of the hands. The authors suggested autosomal recessive transmission of TRPSI, because the unrelated parents of the siblings were unaffected. ${ }^{9}$

We report on a patient with a heterozygous mutation in the TRPS1 gene (c.2735G > A, P.Cys912Tyr). This mutation is not yet described in the literature, but has been considered to be causative, because it involves an amino-acid region that is highly conserved among species. Molecular analysis showed that the same mutation was present in the mother, in the form of somatic mosaicism estimated at $10 \%$ in maternal blood-derived DNA using next generation sequencing (Figure 2). To our knowledge, this is the first description of a mosaic somatic mutation in the TRPS1 gene. Mosaic somatic mutations have been described in many other autosomal dominant or X-linked conditions and have explained the recurrence of disease in siblings born from healthy parents. ${ }^{19}$

Based on this observation, germinal mosaicism is the most likely explanation for recurrences in siblings born of healthy parents, and the autosomal recessive mode of inheritance described in the literature seems unlikely. These data lead us to suggest modifying the genetic counseling for TRPSI. We proposed a $5-10 \%$ risk of recurrence of TRPS1 for healthy parents with an affected child, based on the known recurrence risk in other dominant conditions with demonstrated germinal mosaicism such as that observed in osteogenesis imperfecta, neurofibromatosis type 1 and Kniest dysplasia. ${ }^{20}$ This recurrence risk might concern about half of the TRPSI patients thought to have a de novo mutation. ${ }^{10-13}$
We conclude that TRPSI is inherited in an autosomal dominant fashion, and that the autosomal recessive mode of inheritance proposed to explain recurrence in siblings born of healthy parents is in reality, inheritance through germinal mosaicism, thus changing de facto, the genetic counseling.

\section{CONFLICT OF INTEREST}

The authors declare no conflict of interest.

\section{ACKNOWLEDGEMENTS}

We thank the patient and his family.

1 Klingmuller G: Uber eignetumliche Konstitutions-anomalien der zwei Schwestern und ihre Beziehungen zu neueren entwicklungspathologischenBefunden. Hautarzt 1956; 7: 105-113.

2 Giedon A: Das tricho-rhino-phalangeal syndrome. Helv Paediatr Acta 1966; 21: 475-482.

3 Giedon A: Cone-shaped epiphyses of the hands and their diagnostic value. The tichorhino-phalangeal syndrome. Ann Radiol 1967; 10: 322-329.

4 Niikawa N, Kamei T: The Sugio-Kajii syndrome, proposed tricho-rhino-phalangea syndrome type III. Am J Med Genet 1986; 24: 759-760.

5 Momeni P, Glockner G, Schmidt $O$ et al: Mutations in a new gene, encoding a zincfinger protein, cause tricho-rhino-phalangeal syndrome type I. Nat Genet 2000; 24: 71-74.

6 Napierala D, Sam K, Morello R et al: Uncoupling of chondrocyte differentiation and perichondrial mineralization underlies the skeletal dysplasia in tricho-rhino-phalangeal syndrome. Hum Mol Genet 2008; 17: 2244-2254.

7 Van der Werff ten Bosch JJ: The syndrome of brachymetacarpal dwarfism ("pseudopseudo-hypoparathyroidism') with and without gonadal dysgenesis. Lancet 1959; 1: 69-71, cases 5 and 8 .

8 Hussels IE: Trichorhinophalangeal syndrome in two sibs. Birth Defects Orig Artic Ser 1971; 7: 301-303.

9 Vora NS, Shah S, Dave JN et al: Trichorhinophalangeal syndrome type I. Indian J Dermatol Venereol Leprol 1995; 61: 111-112.

10 Hilton MJ, Sawyer JM, Gutierrez L et al: Analysis of novel and recurrent mutations responsible for the tricho-rhino-phalangeal syndromes. J Hum Genet 2002; 47: 103-106. 
11 Howell CJ, Wynne-Davies R: The tricho-rhino-phalangeal syndrome: a report of 14 cases in 7 kindreds. J Bone Joint Surg Br 1986; 68: 311-314.

12 Ludecke HJ, Schaper J, Meinecke P et al: Genotypic and phenotypic spectrum in tricho-rhino-phalangeal syndrome type I and III. Am J Hum Genet 2001; 68: 81-91.

13 Chen LH, Ning CC, Chao SC: Mutations in TRPS1 gene in trichorhinophalangeal syndrome type I in Asian patients. Br J Dermatol 2010; 163: 416-419.

14 Beals RK: Tricho-rhino-phalangeal dysplasia: report of a kindred. J Bone Joint Surg Am 1973; 55A: 821-826.

15 Besser F, Wells RS: The tricho-rhino-phalangeal syndrome (a family with three affected members). Br J Dermatol, 95: 1976; suppl 14 39-41.
16 Felman AH, Frias JL: The tricho-rhino-phalangeal syndrome: study of 16 patients in one family. AJR Am J Roentgenol 1977; 129: 631-638.

17 Ferrandez A, Remirez J, Saenz P et al: The tricho-rhino-phalangeal syndrome: report of 4 familial cases belonging to 4 generations. Helv Paediatr Acta 1980; 35: 559-567.

18 Giedion A, Burdea M, Fruchter Z et al: Autosomal dominant transmission of the trichorhino-phalangeal syndrome: report of 4 unrelated families, review of 60 cases. Helv Paediatr Acta 1973; 28: 249-259.

19 Erickson RP: Somatic gene mutation and human disease other than cancer: an update. Mutat Res 2010; 705: 96-106.

20 Zlotogora J: Germ line mosaicism. Hum Genet 1998; 102: 381-386. 